\title{
9 \\ Social engagement in late life
}

\section{Tim D Windsor, Rachel G Curtis and Mary A Luszcz}

Gerontologists and older adults agree that remaining socially engaged into late life represents a hallmark of ageing well. It is now well established that having diverse, supportive social networks can promote physical and mental health. Social network members can have a positive influence on health behaviours (e.g. a friend or relative encouraging exercise or medication adherence), provide support during times of need, and allow opportunities for social interactions that create positive emotional experiences (Berkman 1995; Thoits 2006). The past decade has also seen increasing research interest in the possibility that social engagement protects against cognitive decline and dementia (e.g. Fratiglioni et al. 2004; Hertzog et al. 2008; Windsor et al. 2014). Individuals who remain active and socially engaged across the retirement transition and into late life are also best placed to continue making a strong social and economic contribution; a factor likely to prove critical in successfully navigating some of the challenges of population ageing.

This chapter draws on theory and empirical research from social gerontology and life-span developmental psychology to outline a number of key factors that influence older adults' social engagement (Bowling and Dieppe 2005; Rowe and Kahn 1987). Where appropriate, we supplement discussion of international research with our own findings from the Australian Longitudinal Study of Ageing (ALSA; Luszcz et al. 2014) to provide a uniquely Australian perspective on 
key factors that influence social activity in late life. Finally, we discuss issues of relevance to public policy, outlining intervention programs designed to reduce social isolation in older adults, discussing the economic contribution made by older adult volunteers, and noting the challenges and opportunities associated with promoting social engagement among oldest-old adults who may be experiencing more significant health and cognitive limitations.

\section{What predicts social engagement among older adults?}

International studies have consistently shown that social networks tend to become smaller, and social engagement declines with advancing age (Lang and Carstensen, 1994). These findings are mirrored in the Australian context, as shown in Figure 1, which displays results of analysis using the ALSA. ${ }^{1}$ Here we see that older participants report lower levels of social activity engagement, and show a steeper average rate of decline in social activity engagement over 18 years.

Researchers have tended to explain declines in social engagement with advancing age in terms of either ageing-related changes in resources, or social motivation (Windsor et al. 2011). Resource-based explanations tend to focus on how changes in one's repertoire of abilities or external 'assets' may have flow-on effects on the nature or extent of social activities or participation. They acknowledge that shrinking networks in later life are in part due to the loss of network members that occurs through the death of family and friends, and also focus on how declining health and mobility (including restricted access to private and public transport) can adversely affect social activity (e.g. Pinquart 2003).

1 Estimates used to generate Figure 1, as well as additional findings based on the ALSA that are reported subsequently, are based on a series of multi-level growth models that examined associations of age, gender, partner status, physical and cognitive health, socioeconomic status, positive affect, and subjective life expectancy with levels, and rates of change in social activity. Interested readers are invited to contact the first author for details of the analysis and results. 


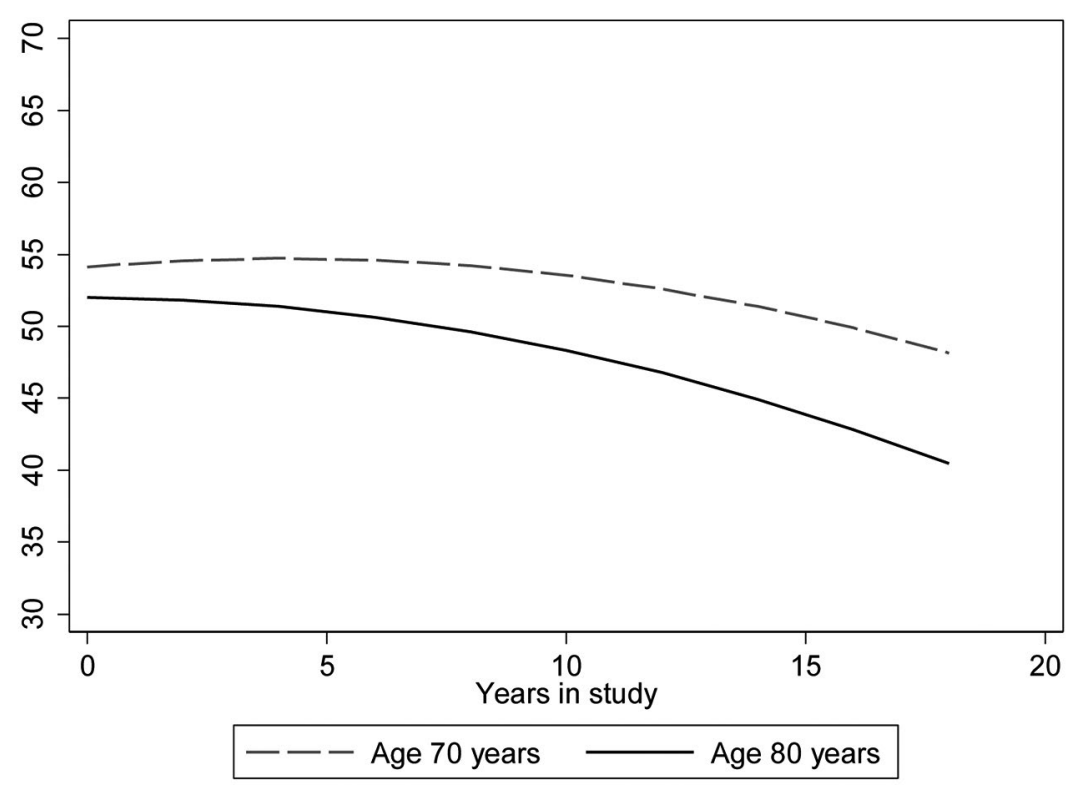

Figure 1. Social activity level change age as people grow older, Australian Longitudinal Study of Ageing

Estimated average trajectories of change in social activity for ALSA participants aged 70 , and aged 80 at baseline. Older adults showed lower initial levels of social activity engagement, and this age differential increased in size over time.

Source: Provided by author.

Surviving to the 'fourth age' or oldest-old adulthood (e.g. ages 85 and above) might be regarded as a major achievement in and of itself. However, whereas the third age for many is a period of vitality and engagement, the fourth age is more typically accompanied by significant declines in health and functioning (Baltes and Smith 2003). In turn, losses in mobility, energy, and cognitive and sensory functioning may adversely affect levels of out-of-home activity and social contact among the oldest-old (Pinquart 2003). Various empirical studies have linked poor health with reduced social activity among older adults. For example, a population-based study of adults aged 57-85 found that those who were more socially disconnected also reported poorer health (Cornwell and Waite 2009). Complementing this finding, another cross-sectional study of adults aged 65-85 indicated that social connectedness (but not social support availability) was associated with better self-reported health (Ashida and Heaney 2008). 
Cognitive health is also likely to be an important resource in driving levels of social activity engagement in older adulthood. To date, research has tended to focus on the extent to which maintaining levels of social engagement provides opportunities for cognitive enrichment that could help to maximise cognitive performance potential (e.g. Hertzog et al. 2008). However, it is also likely that normal and pathological cognitive ageing curtails social engagement. For example, dementia (particularly Alzheimer's disease) is preceded by a long pre-clinical phase characterised by milder cognitive deficits believed to reduce capacity around activities of daily living as well as precipitating withdrawal from social and leisure activities (Stoykova et al. 2011). The importance of health and cognition as enablers of late life social engagement is supported by results of our ALSA analysis, which showed that measures of disability and cognitive impairment were robust predictors of levels of activity engagement after controlling for a range of sociodemographic and individual difference (positive affect, subjective life expectancy) variables.

In addition to ageing-related changes in health and cognitive capacity, sociostructural factors such as social disadvantage are also relevant to social engagement. Theoretical perspectives, concerned with the link between socioeconomic status (SES) and health, recognise social disadvantage as resulting in fewer opportunities to accumulate the economic, behavioural and psychosocial resources believed to protect against health risk (e.g. Gallo 2009). For example, financial hardship may necessitate living in disadvantaged neighbourhoods where levels of social cohesion, trust, and reciprocity are low (Chen and Miller 2013). Low neighbourhood cohesion might be particularly disadvantageous to older adults living alone and in poor health, who might otherwise rely on their neighbours for support (Laporte et al. 2008).

Different structural opportunities established early in life might also have implications for the establishment and maintenance of social networks throughout adulthood. For example, individuals with higher educational attainment may have greater opportunities to develop communication, cognitive, and social skills, which in turn facilitate forming and maintaining relationships (Broese Van Groenou and Van Tilburg 2003). Empirical research generally supports the premise that, among older people, those with low SES report smaller networks with fewer non-kin relationships relative to those with higher SES (e.g. Antonucci 2001; Smith and Baltes 1998; Wenger 1995). 
For example, a large study of older Dutch adults found that low SES older adults reported smaller networks, and lower levels of support from non-kin, relative to those with high SES (Broese Van Groenou and Van Tilburg 2003), and our own analysis of ALSA points to those who are better educated engaging in more social activity.

Explanations that focus on motivation for decreasing social engagement recognise the extent to which older adults play a deliberate role in shaping their own social development. According to socioemotional selectivity theory (SST; Carstensen and Lockenhoff 2003), recognition of one's mortality, or limits to time remaining, become more salient with advancing age, and this results in a re-prioritisation of goals. Specifically, future-oriented goals (e.g. acquiring knowledge to create opportunities) that are well served by larger, more diverse social networks are replaced by presentoriented goals (e.g. maximising the quality of emotional experiences in the here and now) that are better served by smaller, more close-knit networks. Thus, older adults may reduce their levels of engagement with more peripheral social network members in order to spend their remaining time with those who are most important to them.

Ageing-related changes in motivation and social behaviour described by SST have been invoked to account for the better quality of emotional experience frequently reported by older, relative to younger, adults (e.g. Charles and Carstensen 2009; Windsor et al. 2011), and the findings that older adults typically report smaller social networks, but at the same time greater satisfaction with their networks (Lang and Carstensen 1994). Consistent with SST, in the ALSA, perceptions of more time remaining to live are associated with greater social activity engagement; however, this relationship is accounted for to a large degree by individual differences in disability and cognitive limitations. Thus, the ALSA findings provide some broad support for SST, but also suggest that both perceived limits to time remaining and decreases in social engagement could result from underlying declines in functional and cognitive capacities.

Our brief coverage of the relevant literature above highlights the broad range of factors that facilitate, or inhibit older adults' social engagement. Among these various influences, physical health and cognitive capacity are central. This is important from a policy perspective, as it suggests that successful public health interventions 
designed to promote ageing well (see Chapter 7, this volume) and cognitive health (see Chapter 8 , this volume) are likely to offer positive flow-on effects for social engagement. There is also, however, a growing interest in the potential benefits of intervention programs designed to directly address social isolation among older adults. In the next section, we provide an overview of such programs, and outline contemporary thinking regarding how interventions for reducing social isolation might be best designed and implemented into the future.

\section{Programs for reducing social isolation among older adults}

Numerous intervention programs with diverse characteristics have been employed worldwide in an effort to reduce social isolation in older adults. Evidence to date indicates that the most effective programs are those that have a theoretical basis, those that include an educational component (e.g. health-related education), and those including social support or activities that target specific groups (e.g. women, caregivers, widowed: Cattan et al. 2005; Dickens et al. 2011). For example, a group-based friendship program offered women weekly lessons over 12 weeks to help them clarify their expectations regarding relationships, develop their interpersonal skills (e.g. listening, self-disclosure), evaluate the potential of their existing friendships to fulfil their relationship needs, and develop plans to achieve personal relationship goals. Participants reported increased friendships and reduced loneliness during the following year (Stevens 2001). Evidence supporting the effectiveness of one-toone interventions, such as home-based visits and telephone support services, is less compelling (Cattan et al. 2005; Dickens et al. 2011; Findlay 2003).

There has been relatively little research on service-oriented and community development approaches to intervention (Cattan et al. 2005). Although researchers have implemented and evaluated a range of interventions for social isolation, limitations in study design have often led to inconclusive findings (Findlay 2003). For example, many studies are characterised by poorly developed interventions, high attrition rates, small or unrepresentative samples, and failure to consider confounding variables (Dickens et al. 2011). Reviews 
of intervention projects are consistent in emphasising the need for better-designed, more rigorous approaches to evaluation (Cattan et al. 2005; Dickens et al. 2011; Findlay 2003).

A research-to-practice consensus workshop concerned with advancing research on interventions for social isolation among older adults outlined five practice-based priorities (Sabir et al. 2009):

- A first priority was concerned with shedding light on barriers to service utilisation and ways to increase participation among older adults who do not access free services.

- Second, emphasis was placed on the need to develop an easily administered measure for the identification of older adults at risk of social isolation. Positive outcomes of effective interventions may have been obscured in previous studies through a focus on general populations of older adults assumed to be experiencing isolation, rather than targeting those genuinely isolated (Dickens et al. 2011).

- A third priority concerned the implementation of more effective means of evaluating one-to-one direct and indirect contact interventions. This is important for older adults who face barriers to participation in group interventions due to, for example, physical frailty. One-to-one interventions may prove to be more effective than has been previously established when implemented with a more rigorous research design and approach to evaluation.

- Fourth, the importance of designing and evaluating interventions that address multiple possible causes of social isolation was identified. For many older adults, social isolation could arise from the cumulative effects of multiple risk factors (e.g. poor health, limited transport options, and language barriers); however, the effectiveness of multicomponent interventions designed to address manifold challenges is yet to be established.

- A final priority was concerned with research focusing on how provision of services promoting social engagement needs to be effectively balanced with the rights of older adults to make their own decisions regarding the services they access. Part of this focus on 'self-determination' was also concerned with the value of promoting reciprocity in relationships, whereby older adults have the opportunity to make an active contribution to the wellbeing of others, rather than simply being passive recipients of services (Sabir et al. 2009). 
Several Australian-based researchers, policymakers, and organisations are at the forefront of efforts aimed at reducing social isolation among older adults. This is perhaps best illustrated by the Queensland Cross-Government Project to Reduce Social Isolation in Older People (CGPRSIOP: Bartlett et al.2013). Through literature reviews, community consultations, and the evaluation of multiple demonstration projects, the CGPRSIOP developed a set of best-practice guidelines for reducing social isolation (Department of Communities 2009). A number of these guidelines echo key recommendations and priorities from the international perspectives outlined above (Cattan et al. 2005; Sabir et al. 2009), including the need for theoretically based programs with a built-in evaluation component, the value in targeting high-risk groups, the importance of programs offering meaning and purpose to participants, and the benefits of engaging older adults in the design and implementation of programs for reducing social isolation.

Guidelines from the CGPRSIOP study also emphasise the need for a whole-of-community response, involving collaboration and information sharing across government and non-government sectors, to effectively address the wide array of factors that can contribute to social isolation. One such factor that is worth highlighting is access to appropriate, safe, and affordable transport, which is essential for the equitable access of older adults to programs and services. A group who may be at particular risk of isolation are those who have given up driving due to declining health (Marottoli et al. 2000). Finally, the CGPRSIOP argues that the interventions with the greatest potential for long-term success and sustainability are those that build on existing community programs, resources, and organisations.

Pilot programs set up by the CGRSIOP have included physical exercise and education groups, a focus on better service integration and provision with an associated 'buddy system', and a volunteer service providing resources to older adults from culturally and linguistically diverse backgrounds. Initial evaluation of the pilot programs produced mixed findings, and highlighted a number of methodological challenges associated with implementing a rigorous evaluation. These included problems of adequate sample size, sample composition and representativeness, attrition, the difficulty in controlling for changes in social contact outside the scope of the intervention, and problems 
with the standardisation and administration of evaluation procedures across different programs, community, and service-provider groups (Bartlett et al. 2013).

Taken together the evidence to date suggests that the establishment of effective, rigorous, and robust means of evaluating interventions for social isolation among older adults may be the most pressing challenge facing researchers. It is also a critical challenge to overcome, as despite the credibility and likely value of many such programs, it will be difficult to justify substantial investment in the absence of their demonstrated efficacy. Here, we reiterate Findlay's (2003) point that 'networking between communities, governments, the private sector and researchers is essential to provide target-group input, financial support and technical expertise necessary for thorough evaluations of interventions' ( $p$ 655).

Efforts at improving approaches to implementation and evaluation of social isolation interventions may be boosted by a recently funded Australian Research Council Linkage project concerned with analysing the effectiveness of social isolation interventions, and identifying models of social inclusion that have the potential to make the greatest positive impact (see Noone 2011). Although still in its early stages, findings from this research program will have the potential to inform the further development and implementation of new interventions, and/or the expansion of successful existing approaches.

\section{Social engagement and the economic contribution of older Australians}

Maximising opportunities for older Australians to remain actively engaged in their communities is not only critical for the continued wellbeing of individuals and societies, it also represents an important economic goal in the context of population ageing. As outlined in the final report compiled by the Advisory Panel on the Economic Potential of Senior Australians (APEPSA 2011), while projections indicate significant economic challenges arising from population ageing, it is also true that population ageing brings significant economic opportunities. The report advocates a whole-of-government approach to maximising the productive potential of older Australians through 
addressing discriminatory attitudes that restrict opportunities for workforce participation, increasing workplace flexibility, removing barriers to participation that exist in the built environment, and promoting lifestyle choices that enhance population health, and in turn older adults' potential for productive engagement.

Also recognised in the APEPSA report are the substantial social and economic contributions made by older Australians through their participation in volunteering. For example, in South Australia, volunteering was estimated to contribute just under $\$ 5$ billion annually to the state economy during the early to mid-2000s. In 2010, among those volunteering formally for organisations, an estimated 22 per cent of the total value was contributed by adults aged 55-64, with 25 per cent contributed by those aged 65 and older (Ironmonger 2011). Of additional significance is the well-established finding that volunteering is of benefit not just to society and the economy, but also to those who volunteer. Volunteering provides a formal social role that can replace previous work-based roles post-retirement (Greenfield and Marks 2004), and studies have linked volunteering among older adults to a range of beneficial outcomes including better health, reduced depressive symptoms, increased life satisfaction, and longevity (Morrow-Howell 2010).

The potential for volunteering to offer a salubrious 'double-whammy' means that identifying opportunities to facilitate volunteering for those with the interest and capacity, and removing barriers to volunteering, represent topics of keen interest to researchers and policymakers. Australian research (Warburton et al. 2007) reveals a need for more effective promotion of volunteering among older adults, including opportunities for training, flexibility of hours, and education for the latent volunteer population, which highlights the fact that volunteering opportunities exist for those with a diverse array of circumstances, needs, and skills.

The APEPSA (2011) also point to concerns regarding personal liability as a potential barrier to older adults' volunteering. They go on to recommend a review of legislation, and discussions involving government and industry regarding the availability of appropriate and affordable insurance cover for older volunteers. Facilitating volunteering opportunities for older adults with a diverse array of backgrounds and skills is likely to be at the centre of future efforts to enhance social participation among older adults. To this end, 
continuing to effectively link older adults with existing volunteer organisations will be a central component. In addition, flexible and creative thinking concerned with harnessing the potential of older Australians to contribute and remain engaged in novel ways represents an exciting future challenge.

\section{Social engagement among the oldest-old}

Much of our discussion so far regarding social engagement among older Australians rests on assumptions of older adults ageing well (see Chapter 7, this volume), retaining independence, and remaining relatively free of significant physical and cognitive impairment during the post-retirement years. As outlined above, good health and cognition are key resources for social engagement, and health promotion efforts aimed at compression of morbidity, and delay of cognitive decline and dementia (see Chapter 8 , this volume) are likely to provide downstream benefits for social and productive engagement at the population level. However, as individuals progress into oldestold adulthood (or the 'fourth age'), more significant ageing-related losses become normative (Baltes and Smith 2003). Consequently, a challenge for public policy, service providers, and communities, concerns how to best provide opportunities for older adults to remain socially engaged in the context of more serious biological limitations.

This challenge is touched upon in a review of evidence concerned with loneliness and isolation among older adults in residential care (Grenade and Boldy 2008). The transition to care may result in a disruption to existing networks and a reduced frequency of contact with family and friends. At the same time, some older adults (particularly those previously living alone) may experience an increase in social contact through day-to-day interactions with staff and other residents. Important considerations for fostering positive relationships among those in residential care include appropriate staff training and retention policies, and providing opportunities for older residents to exercise independence, and promote feelings of autonomy and selfesteem. According to Grenade and Boldy, while evaluation evidence is scarce, interventions aimed at promoting contact among residents in family- and pet-friendly aged-care environments are likely to provide benefits for social connectedness. 
The issue of social isolation among older adults with more significant health restrictions is also addressed in a recent briefing paper from the Social Policy Research Centre in partnership with the Benevolent Society (2009), which identified practice implications for community aged-care providers. In line with a number of the strategies for reducing social isolation in the community discussed above, the paper identified the importance of strategic approaches that target specific groups, and involve older adults themselves in program planning, delivery and evaluation. Combining social isolation programs with broader multifaceted approaches to health promotion is also recommended. Another important theme concerned the development of programs that enhance the autonomy of older participants by focusing on their strengths and achievements, facilitating the identification of goals, and building self-worth and confidence.

George and Singer (2011) recently described an innovative intervention involving older adults with mild to moderate dementia participating in a volunteer program that involved mentoring kindergarten children in educational activities in a classroom setting. Results of a small, randomised controlled trial indicated that participants in the intervention typically showed better outcomes in stress, cognition, and sense of purpose relative to a control group. Only group differences in stress reached statistical significance; however, moderate effect sizes evident for other quality of life outcomes suggested that this was a result of small sample size (intervention $n=8$; control group $n=7$ ). The role of the intervention in improving outcomes for participants was also supported by qualitative ethnographic methods that suggested perceived benefits for health, purpose and quality of relationships (George 2011). Intergenerational programs of this type hold significant promise as a means of enhancing quality of life for healthy as well as more vulnerable older adults, and for building more cohesive communities by reducing negative aged-based stereotypes. We return to the discussion of intergenerational programs in the concluding section. 


\section{Summary and conclusions}

Either by choice, or necessity, the nature of our social relationships tends to change with age. In this chapter, we outlined some of the main factors thought to influence such changes, before discussing approaches to interventions designed to reduce social isolation, the relevance to society and the economy of older adults' social participation in the form of work and volunteering, and the challenges of promoting social engagement and inclusion among the oldest-old. Here, we conclude by reiterating several key points, and by introducing some additional issues for researchers and policymakers to consider.

An important theme that should inform future refinement and development of public policy related to older adults' social engagement concerns how different generations can work together to contribute to, and benefit from, broader approaches to social inclusion. As touched on above, older adults themselves represent a heterogeneous group, with the vitality and productive engagement of many older adults in the third age (e.g. ages 65-84) standing in contrast to the relatively greater dependency and health restrictions of many in the fourth age (e.g. ages 85 and older; Baltes and Smith 2003). Population ageing will see an increase in both of these age groups, and creative approaches to social engagement might focus on how volunteering efforts of the young-old can best support the social inclusion and autonomy of the oldest-old.

Recent years have seen an increased focus on the value of social initiatives and aspects of urban design (e.g. Eheart et al. 2009) that bring together children and/or younger adults with older adults. Such intergenerational programs are concerned with promoting interaction and cooperation among different generations in ways that promote attitudinal change and build social capital (Cummings et al. 2002). Research based on intergenerational programs has revealed promising findings including better school behaviour (Cummings et al. 2002) and reduced ageist stereotypes (Cummings et al. 2002; Pinquart et al. 2000) among child participants, and better perceptions of neighbourhood cohesion among older adults (de Souza and Grundy 2007). However, as is the case with interventions for social isolation, the wider application of intergenerational programs has been restricted by a lack of research evidence regarding their effectiveness (Statham 2009). 
A notable exception is the US-based Experience Corps ${ }^{\circledR}$ (EC) program, which recruits older adult volunteers to assist in schools, providing support in programs designed to improve student literacy and behaviour. The EC program is founded on a number of specific elements designed to enhance its broad effectiveness in improving outcomes for students, older volunteers, and the community (Glass et al. 2004). For example, volunteers are required to make a substantial time commitment to the program, which is designed to foster continuity of volunteer-student relationships, and maximise opportunities for health and cognitive benefits for older adults arising from their volunteer activity. The program also offers training and infrastructure support for participants, as well as modest reimbursement for participants' expenses, thereby reducing barriers to participation among those on lower incomes. The EC program also targets schools and volunteers from lower socioeconomic areas, contributing to social capital where it is most needed.

An initial evaluation of the pilot EC program was based on a randomised controlled trial, and pointed to beneficial outcomes for volunteers in terms of aspects of cognitive test performance, physical activity, and social support (Rebok et al. 2011). Its well-developed conceptual basis and the rigorous approach to evaluation that underlies the EC program means that it provides a useful model to draw on in the design of future intergenerational programs in Australia.

As we have emphasised, social connections offer wide-ranging benefits for the health and wellbeing of individuals, and societies. However, we would also add a cautionary note that those concerned with efforts to enhance social participation and engagement among older adults should be mindful of the potential for social relationships to result in tension and conflict as well as positive outcomes. A growing body of research now demonstrates that negative social exchanges are a significant source of stress, and can adversely impact health and wellbeing. Moreover, the detrimental effects of negative social exchanges on wellbeing may be stronger than the positive effects resulting from supportive relationships (Newsom et al. 2005; Rook 2015). Thus, the most effective interventions may be those that enhance engagement and support among older adults while also providing a means for participants to readily disengage from relationships that they see as problematic. 
To conclude, physical and cognitive health are important resources for social engagement in older adulthood. As a result, public health interventions designed to promote physical and cognitive health over the life-span are likely to have flow-on benefits for the social engagement of older adults. In addition, interventions that directly target social isolation among older adults show substantial promise; however, better approaches to evaluation are needed to identify those interventions most likely to be efficacious for different at-risk groups, both in the community and in residential care settings. Researchers have emphasised that the inclusion of older adult target groups in all stages of program design and evaluation is likely to be essential for the development of successful interventions for social isolation.

The design of interventions should also ensure that the social connections being fostered, wherever possible, allow older adults to maintain and develop a sense of autonomy, purpose, and meaning around their activities; intergenerational programs appear to hold considerable promise as a means of achieving these goals. Older adults represent a heterogeneous group, with great variation in both capacities for, and individual inclinations toward, broader social engagement. As a result, social engagement and inclusion is likely to be best promoted through multifaceted and multidisciplinary efforts. Promoting healthy ageing and addressing issues of social disadvantage are likely to be central to these endeavours.

Ultimately, removing barriers to social engagement among older adults stands to create more cohesive communities, and to maximise the economic and social contribution of older Australians in the context of population ageing.

\section{References}

Advisory Panel of the Economic Potential of Senior Australians (APEPSA) (2011). Realising the economic potential of senior Australians: Turning grey into gold. Canberra: Commonwealth of Australia.

Antonucci Toni (2001). Social relations: An examination of social networks, social support and sense of control. In James E Birren and Klaus Warner Schaie (Eds) Handbook of the Psychology of Aging (5th ed). New York: Academic Press, pp. 427-453. 
Ashida Sato and Heaney Catherine (2008). Differential associations of social support and social connectedness with structural features of social networks and the health status of older adults. Journal of Aging and Health, 20: 872-893.

Baltes Paul and Smith Jacqui (2003). New frontiers in the future of aging: From successful aging of the young old to the dilemmas of the fourth age. Gerontology, 49: 123-135.

Bartlett Helen, Warburton Jeni, Lui Chi-Wai, Peach Linda and Carroll Matthew (2013). Preventing social isolation in later life: Findings and insights from a pilot Queensland intervention study. Ageing and Society, 33: 1167-1189.

Berkman Lisa (1995). The role of social relations in health promotion. Psychosomatic Medicine, 57: 245-254.

Bowling Ann and Dieppe Paul (2005). What is successful ageing and who should define it? BMJ, 331: 1548-1551.

Broese Van Groenou Marjolein and Van Tilburg Theo (2003). Network size and support in old age: Differentials by socio-economic status in childhood and adulthood. Ageing and Society, 23: 625-645.

Carstensen Laura and Lockenhoff Corinna (2003). Aging, emotion, and evolution: The bigger picture. Annals of the New York Academy of Sciences, 1000: 152-179.

Cattan Mima, White Martin, Bond John and Learmouth Alison (2005). Preventing social isolation and loneliness among older people: A systematic review of health promotion interventions. Ageing and Society, 25: 41-67.

Charles Susan and Carstensen Laura (2009). Social and emotional aging. Annual Review of Psychology, 61: 383-409.

Chen Edith and Miller Gregory (2013). Socioeconomic status and health: Mediating and moderating factors. Annual Review of Clinical Psychology, 9: 723-749.

Cornwell Erin York and Waite Linda (2009). Social disconnectedness, perceived isolation, and health among older adults. Journal of Health and Social Behavior, 50: 31-48. 
Cummings Sherry, Williams Mona and Ellis Rodney (2002). Impact of an intergenerational program on 4th graders' attitudes toward elders and school behaviors. Journal of Human Behavior in the Social Environment, 6: 91-107.

de Souza Elza Maria and Grundy Emily (2007). Intergenerational interaction, social capital and health: Results from a randomised controlled trial in Brazil. Social Science and Medicine, 65: 1397-1409.

Department of Communities (2009). Cross-government project to reduce social isolation of older people: Best practice guidelines. Brisbane: Department of Communities, Queensland Government.

Dickens Andy, Richards Suzanne, Greaves Colin and Campbell John (2011). Interventions targeting social isolation in older people: A systematic review. BMC Public Health, 11: 647-647.

Eheart Brenda Krause, Hopping David, Power Martha, Mitchell Elissa, and Racine David (2009). Generations of hope communities: An intergenerational neighborhood model of support and service. Children and Youth Services Review, 31: 47-52.

Findlay Robyn (2003). Interventions to reduce social isolation amongst older people: Where is the evidence? Ageing and Society, 23: 647-658.

Fratiglioni Laura, Paillard-Borg Stephanie and Winblad Bengt (2004). An active and socially integrated lifestyle in late life might protect against dementia. The Lancet Neurology, 3: 343-353.

Gallo Linda (2009). The reserve capacity model as a framework for understanding psychosocial factors in health disparities. Applied Psychology: Health and Well-Being, 1: 62-72.

George Daniel (2011). Intergenerational volunteering and quality of life: Mixed methods evaluation of a randomized control trial involving persons with mild to moderate dementia. Quality of Life Research, 20: 987-995.

George Daniel and Singer Mendel (2011). Intergenerational volunteering and quality of life for persons with mild to moderate dementia: Results from a 5-month intervention study in the United States. The American Journal of Geriatric Psychiatry, 19: 392-396. 
Glass Thomas, Freedman Marc, Carlson Michelle, Hill Joel, Frick Kevin, Ialongo Nick and Fried Linda (2004). Experience Corps: Design of an intergenerational program to boost social capital and promote the health of an aging society. Journal of Urban Health: Bulletin of the New York Academy of Medicine, 81: 94-105.

Greenfield Emily and Marks Nadine (2004). Formal volunteering as a protective factor for older adults' psychological well-being. Journal of Gerontology: Social Sciences, 59B: S258-S264.

Grenade Linda and Boldy Duncan (2008). Social isolation and loneliness among older people: Issues and future challenges in community and residential settings. Australian Health Review, 32: 468-478.

Hertzog Christopher, Kramer Arthur, Wilson Robert and Lindenberger Ulman (2008). Enrichment effects on adults cognitive development: Can the functional capacity of older adults be preserved and enhanced? Psychological Science in the Public Interest, 9: 1-65.

Ironmonger Duncan (2011). The economic value of volunteering in South Australia. Adelaide: Office for Volunteers, Government of South Australia.

Lang Frieder and Carstensen Laura (1994). Close emotional relationships in late life: Further support for proactive aging in the social domain. Psychology and Aging, 9: 315-324.

Laporte Audrey, Nauenberg Eric and Shen Leilei (2008). Aging, social capital, and health care utilization in Canada. Health Economics, Policy and Law, 3: 393-411.

Luszcz Mary, Giles Lynne, Anstey Kaarin, Browne-Yung Kathryn, Walker Ruth and Windsor Tim (2014). Cohort Profile: The Australian Longitudinal Study of Ageing (ALSA). International Journal of Epidemiology, 1-10. doi:1093/ije/dyul96.

Marottoli Richard, Mendes de Leon Carlos, Glass Thomas, Williams Christianna, Cooney Jnr Leo and Berkman Lisa (2000). Consequences of driving cessation: Decreased out-of-home activity levels. Journals of Gerontology: Social Sciences, 55: S334-S340.

Morrow-Howell Nancy (2010). Volunteering in later life: Research frontiers. Journal of Gerontology: Social Sciences, 65B: 461-469. 
Newsom Jason, Roo, Karen, Nishishiba Masami, Sorkin Dara and Mahan Tyrae (2005). Understanding the relative importance of positive and negative social exchanges: Examining specific domains and appraisals. Journal of Gerontology: Psychological Sciences, 60B: P304-P312.

Noone Yasmin (2011). Solving social isolation-Successfully. Australian Ageing Agenda. www.australianageingagenda.com.au/2011/06/28/ solving-social-isolation-successfully/.

Pinquart Martin (2003). Loneliness in married, widowed, divorced, and never-married older adults. Journal of Social and Personal Relationships, 20: 31-53.

Pinquart Martin, Wenzel Silka and Sö Rensen Silvia (2000). Changes in attitudes among children and elderly adults in intergenerational group work. Educational Gerontology, 26: 523-540.

Rebok George, Carlson Michelle, Barron Jeremy, Frick Kevin, McGill Sylvia, Parisi Jeanine and Fried Linda (2011). Experience Corps ${ }^{\circledR}$ : A civic engagement-based public health intervention in the public schools. In Paula E Hartman-Stein and Asenath LaRue (Eds) Enhancing cognitive fitness in adults: A guide for use and development of community-based programs. New York: Springer, pp. 469-487.

Rook Karen (2015). Social networks in later life: Weighing positive and negative effects on health and well-being. Current Directions in Psychological Science, 24: 45-51.

Rowe John and Kahn Robert (1987). Human aging: usual and successful. Science, 237: 143-149. doi:10.1126/science.3299702: 2.

Sabir Myra, Wethington Elaine, Breckman Risa, Meador Rhoda, Reid MC and Pillemer Karl (2009). A community-based participatory critique of social isolation intervention research for communitydwelling older adults. Journal of Applied Gerontology, 28: 218-234.

Smith Jacqui and Baltes Margret (1998). The role of gender in very old age: Profiles of functioning and everyday life patterns. Psychology and Aging, 13: 676-695. 
Social Policy Research Centre and the Benevolent Society (SPRC) (2009). Promoting social networks for older people in community aged care. Research to Practice Briefing 2. Sydney: University of New South Wales and the Benevolent Society.

Statham Elaine (2009). Promoting intergenerational programmes: Where is the evidence to inform policy and practice? Evidence and Policy: A Journal of Research, Debate and Practice, 5: 471-488.

Stevens Nan (2001). Combating loneliness: A friendship enrichment programme for older women. Ageing and Society, 21: 183-202.

Stoykova Ralitsa, Matharan Fanny, Dartigues Jean-François and Amieva Hélène (2011). Impact of social network on cognitive performances and age-related cognitive decline across a 20-year follow-up. International Psychogeriatrics, 23: 1405-1412.

Thoits Peggy (2006). Personal agency in the stress process. Journal of Health and Social Behavior, 47: 309-323.

Warburton Jeni, Paynter Jessica and Petriwskyj Andrea (2007). Volunteering as a productive ageing activity: Incentives and barriers to volunteering by Australian seniors. Journal of Applied Gerontology, 26: 333-354.

Wenger G Clare (1995). A comparison of urban with rural support networks: Liverpool and North Wales. Ageing and Society, 15: 59-81.

Windsor Tim, Fiori Katherine and Crisp Dimity (2011). Personal and neighborhood resources, future time perspective, and social relations in middle and older adulthood. Journals of Gerontology Series B: Psychological Sciences and Social Sciences, 67: 423-431.

Windsor Tim, Gerstorf Denis, Pearson Elissa, Ryan Lindsay and Anstey Kaarin (2014). Positive and negative social exchanges and cognitive aging in young-old adults: Differential associations across family, friend, and spouse domains. Psychology and Aging, 29: 28-43. 
This text is taken from Population Ageing and Australia's Future, edited by Hal Kendig, Peter McDonald and John Piggott, published 2016 by ANU Press, The Australian National University, Canberra, Australia. 